Supporting information for

\title{
Dual Optimization in Phase Transfer Catalyzed Synthesis of Dibenzyl Sulfide using Response Surface Methodology (RSM)
}

\author{
Sujit Sen*, Ujjal Mondal, Gaurav Singh \\ Catalysis Research Laboratory, Department of Chemical Engineering, National Institute of \\ Technology, Rourkela - 769008, India
}

*Corresponding author: Tel: +91-9938246590; Fax: +91-661-2462999; Email: sensujit@gmail.om

\section{Table of Content}

1. Table S1: ANOVA for response surface quadratic model for BC conversion

2. Table S2: ANOVA for response surface quadratic model for DBS Selectivity

3. Table S3. Coefficient Estimate with Standard Error and 95\% Confidence Interval for $\mathrm{BC}$ conversion and DBS selectivity

4. General Information on GC-MS

5. Figure S1: GC spectra showing formation of dibenzyl sulfide from reactant benzyl chloride in solvent toluene.

6. Figure S2: Mass spectra for all the peaks shown in Figure S1

7. Figure S3: Mass spectra of product dibenzyl sulfide 
Table S1. ANOVA for response surface quadratic model for BC conversion ${ }^{\mathrm{a}}$

\begin{tabular}{cccccc}
\hline Source & $\begin{array}{c}\text { Sum of } \\
\text { Squares }\end{array}$ & $\begin{array}{c}\text { Degree of } \\
\text { Freedom }\end{array}$ & $\begin{array}{c}\text { Mean } \\
\text { Square }\end{array}$ & F-Value & p-value \\
\hline Model & 2383.22 & 14 & 170.23 & 2932.20 & $<0.0001$ \\
A-Temperature & 281.53 & 1 & 281.53 & 4849.41 & $<0.0001$ \\
B-Catalyst & 147.01 & 1 & 147.01 & 2532.32 & $<0.0001$ \\
Concentration & & & & & \\
C-BC/S mole ratio & 1238.41 & 1 & 1238.41 & 21331.41 & $<0.0001$ \\
D-MEA/S mole & 39.01 & 1 & 39.01 & 672.03 & $<0.0001$ \\
ratio & & & & & \\
AB & 0.49 & 1 & 0.49 & 8.44 & 0.0109 \\
AC & 62.41 & 1 & 62.41 & 1075.00 & $<0.0001$ \\
AD & 1.69 & 1 & 1.69 & 29.11 & $<0.0001$ \\
BC & 21.16 & 1 & 21.16 & 364.48 & $<0.0001$ \\
BD & 28.09 & 1 & 28.09 & 483.85 & $<0.0001$ \\
CD & 166.41 & 1 & 166.41 & 2866.39 & $<0.0001$ \\
A $^{2}$ & 206.49 & 1 & 206.49 & 3556.71 & $<0.0001$ \\
B $^{2}$ & 42.43 & 1 & 42.43 & 730.85 & $<0.0001$ \\
C $^{2}$ & 197.19 & 1 & 197.19 & 3396.52 & $<0.0001$ \\
D $^{2}$ & 3.48 & 1 & 3.48 & 59.96 & $<0.0001$ \\
Residual & 0.87 & 15 & 0.058 & & \\
Lack of Fit & 0.77 & 10 & 0.077 & 3.85 & 0.0748 \\
Pure Error & 0.100 & 5 & 0.020 & & \\
Cor Total & 2384.10 & 29 & & & \\
\hline
\end{tabular}


Table S2. ANOVA for response surface quadratic model for DBS Selectivity ${ }^{\mathbf{b}}$

\begin{tabular}{llllll}
\hline Source & $\begin{array}{l}\text { Sum of } \\
\text { Squares }\end{array}$ & df & $\begin{array}{l}\text { Mean } \\
\text { Square }\end{array}$ & $\begin{array}{l}\text { F } \\
\text { Value }\end{array}$ & p-value \\
\hline Model & 12975.80 & 14 & 926.84 & 89.32 & $<0.0001$ \\
A-Temperature & 80.30 & 1 & 80.30 & 7.74 & 0.0140 \\
B-Catalyst & 333.76 & 1 & 333.76 & 32.16 & $<0.0001$ \\
$\begin{array}{l}\text { Concentration } \\
\text { C-BC/S mole }\end{array}$ & 7381.53 & 1 & 7381.53 & 711.35 & $<0.0001$ \\
ratio & & & & & \\
D-MEA/S mole & 3893.85 & 1 & 3893.85 & 375.24 & $<0.0001$ \\
ratio & & & & & \\
AB & 3.33 & 1 & 3.33 & 0.32 & 0.5794 \\
AC & 0.33 & 1 & 0.33 & 0.032 & 0.8607 \\
AD & 6.63 & 1 & 6.63 & 0.64 & 0.4366 \\
BC & 10.40 & 1 & 10.40 & 1.00 & 0.3326 \\
BD & 28.36 & 1 & 28.36 & 2.73 & 0.1191 \\
CD & 13.88 & 1 & 13.88 & 1.34 & 0.2656 \\
A $^{2}$ & 1.09 & 1 & 1.09 & 0.10 & 0.7508 \\
$B^{2}$ & 512.82 & 1 & 512.82 & 49.42 & $<0.0001$ \\
C $^{2}$ & 573.84 & 1 & 573.84 & 55.30 & $<0.0001$ \\
$D^{2}$ & 427.73 & 1 & 427.73 & 41.22 & $<0.0001$ \\
Residual & 155.65 & 15 & 10.38 & & \\
Lack of Fit & 155.60 & 10 & 15.56 & 1414.52 & $<0.0001$ \\
Pure Error & 0.055 & 5 & 0.011 & & \\
Cor Total & 13131.45 & 29 & & & \\
\hline
\end{tabular}


Table S3. Coefficient Estimate with Standard Error and 95\% Confidence Interval for BC conversion and DBS selectivity

\begin{tabular}{|c|c|c|c|c|c|c|c|c|}
\hline \multirow{3}{*}{ Factor } & \multicolumn{4}{|c|}{ BC Conversion } & \multicolumn{4}{|c|}{ DBS Selectivity } \\
\hline & \multirow{2}{*}{$\begin{array}{l}\text { Coefficient } \\
\text { of Estimate }\end{array}$} & \multirow{2}{*}{$\begin{array}{l}\text { Standard } \\
\text { Error }\end{array}$} & \multicolumn{2}{|c|}{$95 \% \mathrm{CI}$} & \multirow{2}{*}{$\begin{array}{l}\text { Coefficient } \\
\text { of Estimate }\end{array}$} & \multirow{2}{*}{$\begin{array}{l}\text { Standard } \\
\text { Error }\end{array}$} & \multicolumn{2}{|c|}{$95 \%$ CI } \\
\hline & & & High & Low & & & High & Low \\
\hline Intercept & 96.00 & 0.098 & 95.79 & 96.21 & 65.05 & 1.32 & 62.25 & 67.85 \\
\hline $\begin{array}{l}\text { A- } \\
\text { Temperature }\end{array}$ & 3.42 & 0.049 & 3.32 & 3.53 & 1.83 & 0.66 & 0.43 & 3.23 \\
\hline $\begin{array}{l}\text { B-Catalyst } \\
\text { Concentration }\end{array}$ & 2.47 & 0.049 & 2.37 & 2.58 & 3.73 & 0.66 & 2.33 & 5.13 \\
\hline $\begin{array}{l}\mathrm{C}-\mathrm{BC} / \mathrm{S} \text { mole } \\
\text { ratio }\end{array}$ & -7.18 & 0.049 & -7.29 & -7.08 & 17.54 & 0.66 & 16.14 & 18.94 \\
\hline $\begin{array}{l}\text { D-MEA/S } \\
\text { mole ratio }\end{array}$ & 1.27 & 0.049 & 1.17 & 1.38 & 12.74 & 0.66 & 11.34 & 14.14 \\
\hline $\mathrm{AB}$ & 0.18 & 0.060 & 0.047 & 0.30 & 0.46 & 0.81 & -1.26 & 2.17 \\
\hline $\mathrm{AC}$ & 1.98 & 0.060 & 1.85 & 2.10 & 0.14 & 0.81 & -1.57 & 1.86 \\
\hline $\mathrm{AD}$ & 0.33 & 0.060 & 0.20 & 0.45 & 0.64 & 0.81 & -1.07 & 2.36 \\
\hline $\mathrm{BC}$ & 1.15 & 0.060 & 1.02 & 1.28 & -0.81 & 0.81 & -2.52 & 0.91 \\
\hline $\mathrm{BD}$ & 1.33 & 0.060 & 1.20 & 1.45 & -1.33 & 0.81 & -3.05 & 0.39 \\
\hline $\mathrm{CD}$ & 3.23 & 0.060 & 3.10 & 3.35 & 0.93 & 0.81 & -0.79 & 2.65 \\
\hline$A^{2}$ & -2.74 & 0.046 & -2.84 & -2.65 & -0.20 & 0.62 & -1.51 & 1.11 \\
\hline $\mathrm{B}^{2}$ & -1.24 & 0.046 & -1.34 & -1.15 & -4.32 & 0.62 & -5.63 & -3.01 \\
\hline $\mathrm{C}^{2}$ & -2.68 & 0.046 & -2.78 & -2.58 & -4.57 & 0.62 & -5.88 & -3.26 \\
\hline $\mathrm{D}^{2}$ & 0.36 & 0.046 & 0.26 & 0.45 & -3.95 & 0.62 & -5.26 & -2.64 \\
\hline
\end{tabular}

\section{General Information}

a) GC-MS: Quantification of organic phase were performed on GC-FID (Agilent GC 7890B)

by using a capillary column DB-5MS, $2 \mathrm{~m}$ x $3 \mathrm{~mm}$, coupled with flame ionization detector.

The product was further confirmed by GC-MS (Agilent 5977A)

\section{Characterization of the product}




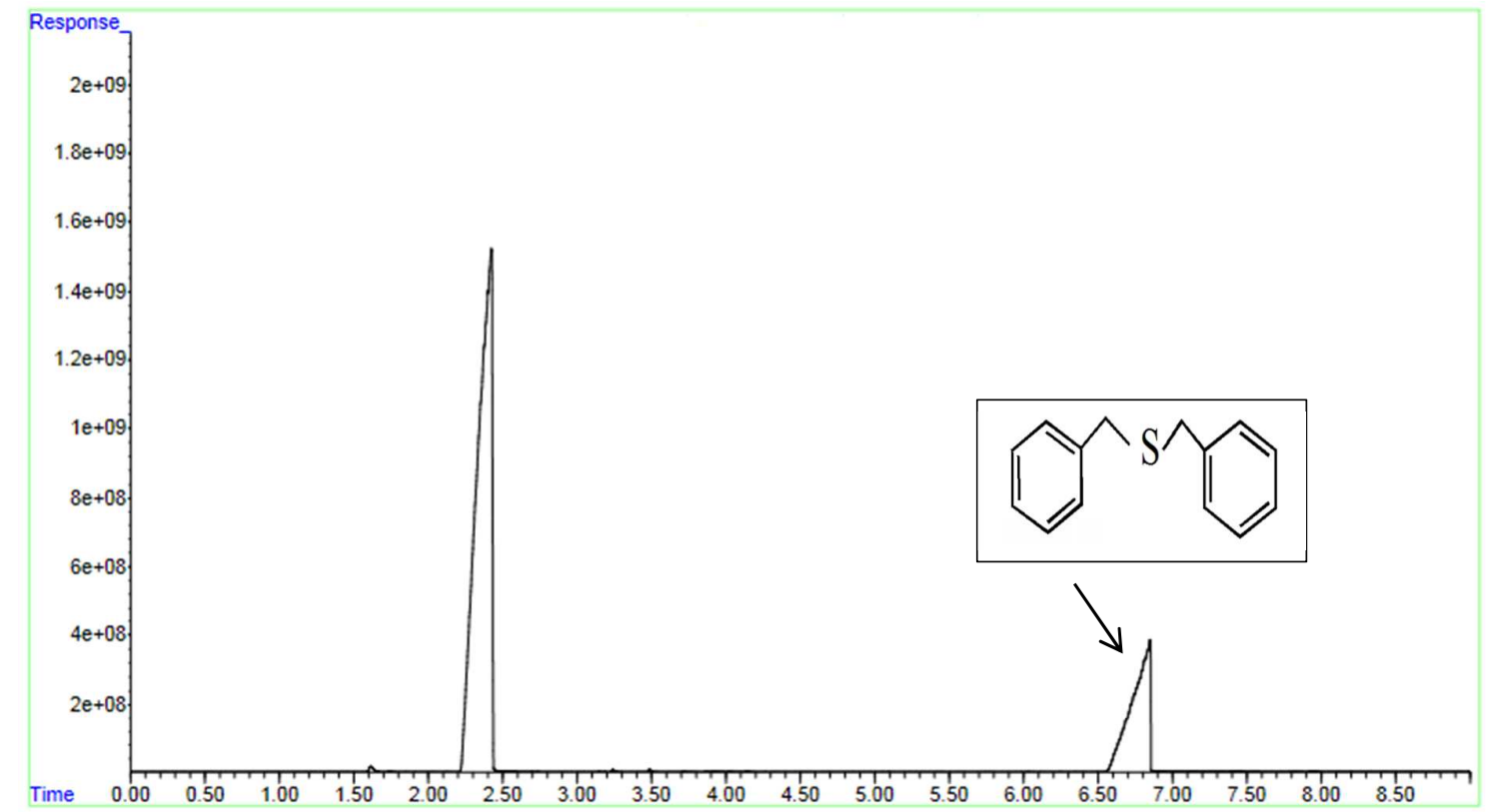

Figure S1. GC spectra showing formation of dibenzyl sulfide from reactant benzyl chloride in solvent toluene. 


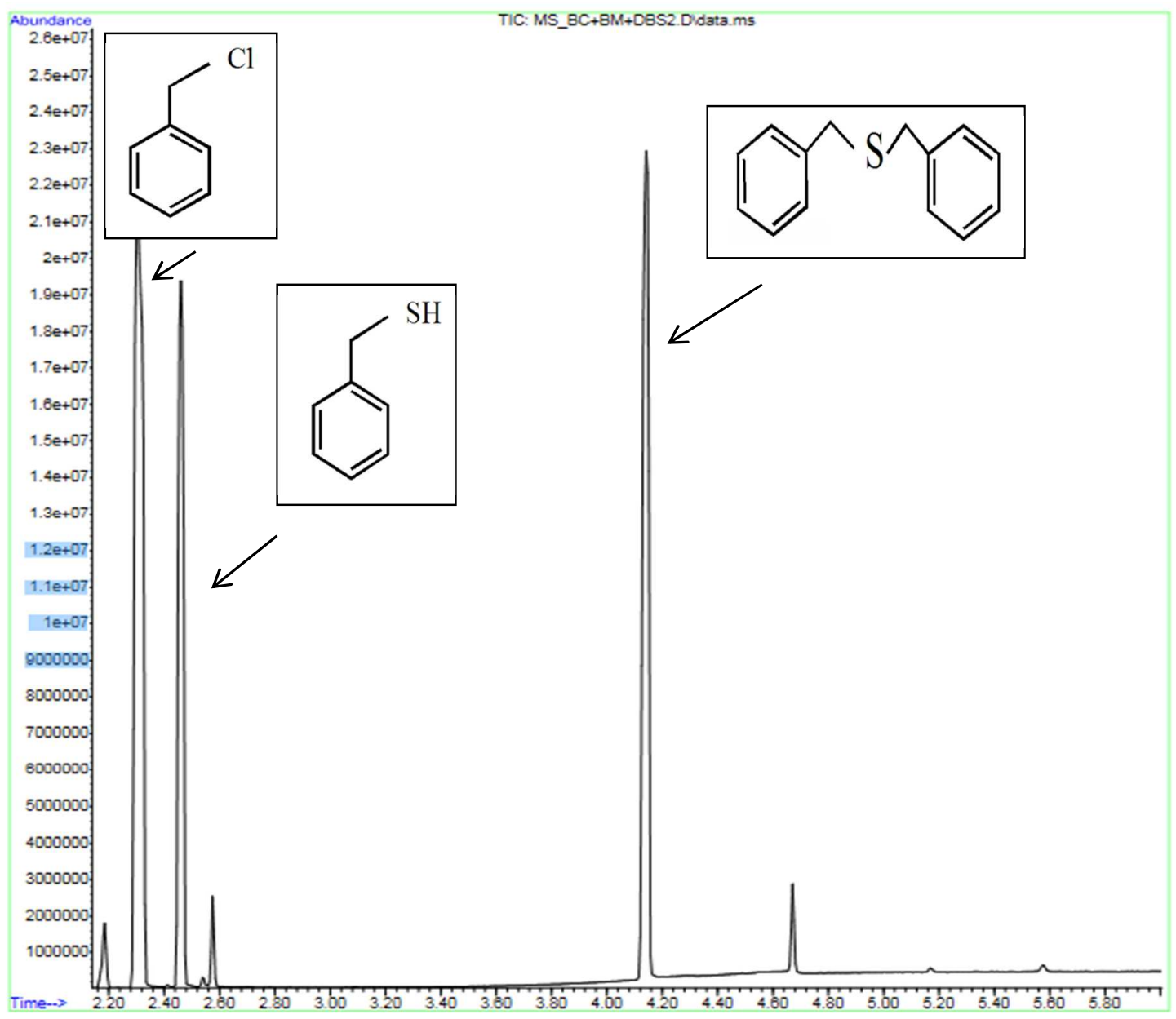

Figure S2. Mass spectra for all the peaks shown in Figure S1 


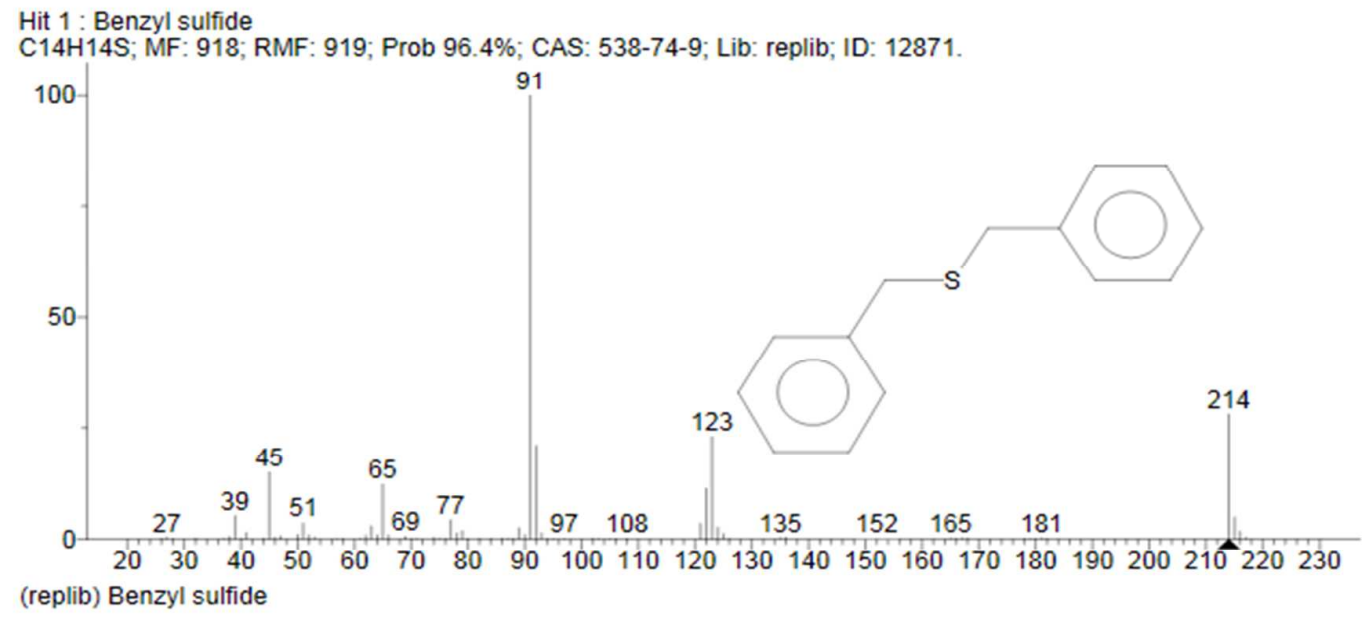

Figure S3. Mass spectra of product dibenzyl sulfide 